\title{
3,4-Bis(3-tetrazolylfuroxan-4-yl)furoxan: A Linear C-C Bonded Pentaheterocyclic Energetic Material with High Heat of Formation and Superior Performance
}

\author{
Lianjie Zhai, ${ }^{\dagger \dagger}$ Fuqiang $\mathrm{Bi}^{\dagger}$, Junlin Zhang, ${ }^{\dagger}$ Jiarong Zhang, ${ }^{\dagger}$ Xiangzhi Li, ${ }^{\dagger}$ Bozhou Wang, ${ }^{*}, \dagger$ and Sanping Chen $*, \dot{*}$ \\ † State Key Laboratory of Fluorine \& Nitrogen Chemicals, Xi’an Modern Chemistry Research Institute, Xi’an, China; \\ E-mail: wbz600@163.com \\ $\$$ College of Chemistry and Materials Science, Northwest University, Xi’an 710127, China. \\ E-mail: sanpingchen@126.com.
}

Table of Contents

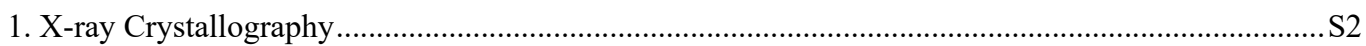

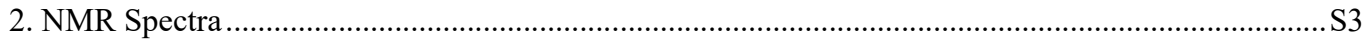

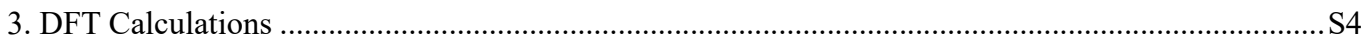

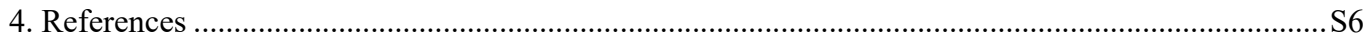




\section{X-ray Crystallography}

Table S1. Crystallographic data for BTTFO $\cdot \mathrm{H}_{2} \mathrm{O}$

\begin{tabular}{|c|c|}
\hline Empirical formula & $\left(\mathrm{C}_{8} \mathrm{H}_{2} \mathrm{~N}_{14} \mathrm{O}_{6}\right)_{2}\left(\mathrm{H}_{2} \mathrm{O}\right)_{2}$ \\
\hline $\mathrm{FW} / \mathrm{g} \mathrm{mol}^{-1}$ & 816.50 \\
\hline Temperature $/ \mathrm{K}$ & 296 \\
\hline Crystal system & Orthorhombic \\
\hline Space group & $P 2_{1} 2_{1} 2_{1}$ \\
\hline Crystal size(mm) & $0.23 \times 0.16 \times 0.14$ \\
\hline$a(\AA)$ & $5.383(3)$ \\
\hline$b(\AA)$ & $12.923(7)$ \\
\hline$c(\AA)$ & $43.54(2)$ \\
\hline$V\left(\mathrm{~nm}^{3}\right)$ & $3.029(3)$ \\
\hline Z & 4 \\
\hline$D_{c}\left(\mathrm{~g} \mathrm{~cm}^{-3}\right)$ & 1.791 \\
\hline$\mu\left(\mathrm{mm}^{-1}\right)$ & 0.158 \\
\hline $\mathrm{F}(000)$ & 1648 \\
\hline$\theta$ range for data collection & 1.87 to 25.24 \\
\hline Dataset $\mathrm{h}$ & $-6 ; 7$ \\
\hline Dataset $\mathrm{k}$ & $-16 ; 17$ \\
\hline Dataset 1 & $-58 ; 42$ \\
\hline Reflections collected & 7195 \\
\hline Independent reflections & 5073 \\
\hline Data/restraints/parameters & $7195 / 0 / 556$ \\
\hline Goodness-of-fit on $F^{2}$ & 0.990 \\
\hline$R_{1}, w R_{2}[I>2 \sigma(I)]$ & $0.0432,0.0899$ \\
\hline$R_{1}, w R_{2}$ & $0.0707,0.993$ \\
\hline Resd. Dens./e $\AA^{-3}$ & 0.244 and -0.185 \\
\hline Absorption correction & multi-scan \\
\hline $\mathrm{CCDC}$ & 1987360 \\
\hline
\end{tabular}



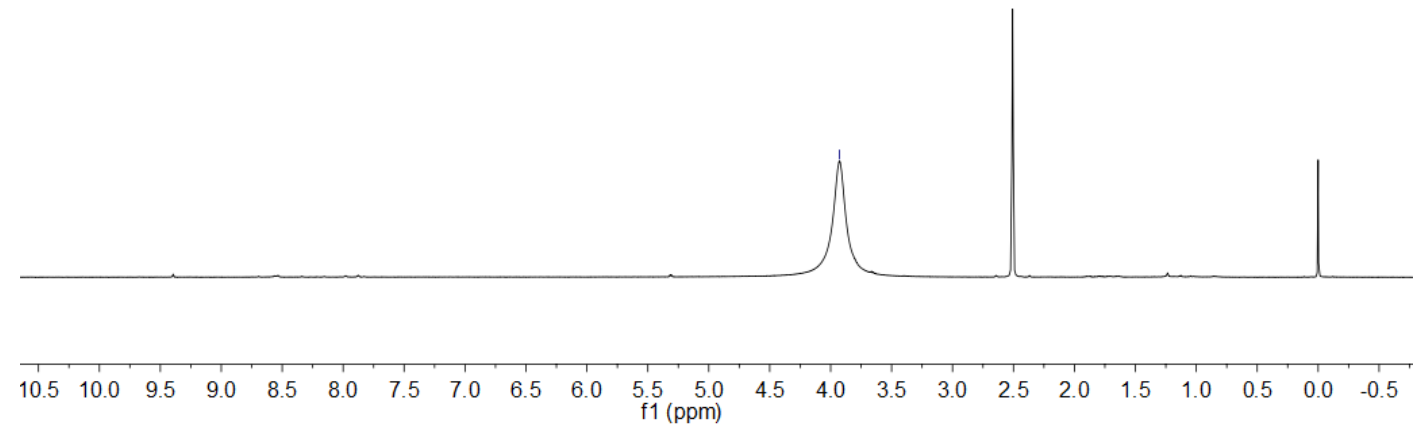

Figure S1. ${ }^{1} \mathrm{H}$ NMR spectrum of BTTFO in $\left[\mathrm{D}_{6}\right] \mathrm{DMSO}$

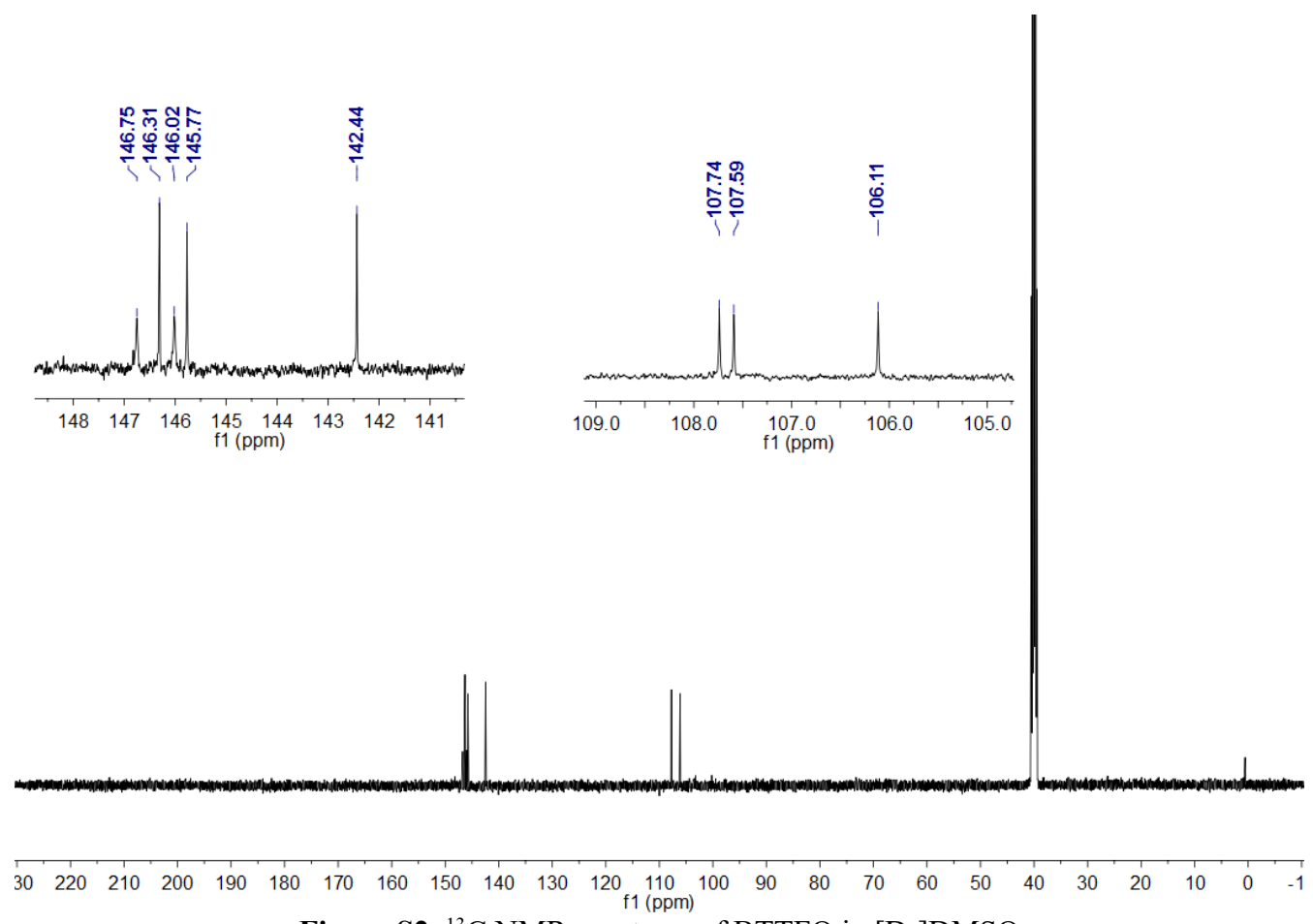

Figure S2. ${ }^{13} \mathrm{C}$ NMR spectrum of BTTFO in $\left[\mathrm{D}_{6}\right] \mathrm{DMSO}$ 


\section{DFT Calculations}

All quantum chemical calculations were carried out using the Gaussian 09 (Revision A.02) program package ${ }^{[1]}$ and visualized by GaussView 5.08. ${ }^{[2]}$ The geometric optimization and frequency analyses of the structures were carried out using the B3LYP functional with $6-31+\mathrm{G}^{* *}$ basis set, ${ }^{[3]}$ and single energy points were calculated at the MP2/6-311++G** 1 evel. ${ }^{[4]}$ All of the optimized structures were characterized to be true local energy minima on the potential energy surface without imaginary frequencies.

The heat of formation was determined using an isodesmic reaction (Figure S3). The heats of formation for furoxan, and tetrazole were obtained by an atomization approach using CBS-4M method ${ }^{[5,6]}$. The heats of formation of $\mathrm{CH}_{4}$ and $\mathrm{CH}_{3} \mathrm{CH}_{3}$ were obtained from the NIST WebBook. ${ }^{[7]}$ Some parameters (E, ZPE, H, HOF) were listed in Table S2.

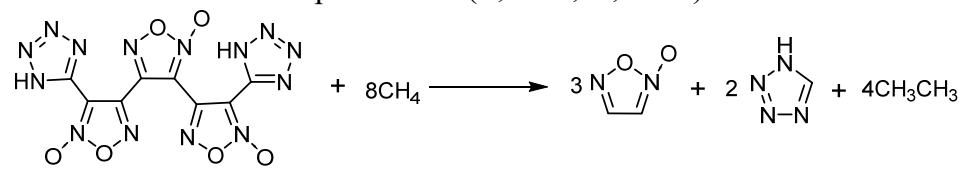

Figure S3. Isodesmic reaction for calculating heat of formation for BTTFO.

Table S2. Calculated total energy, zero-point energy, values of the correction and gas phase heats of

\begin{tabular}{ccccccc}
\hline \multicolumn{7}{c}{ formation (HOF). } \\
Comp. & $\begin{array}{c}\mathrm{ZPE}{ }^{\mathrm{a}} \\
(\mathrm{au})\end{array}$ & $\begin{array}{c}H_{\mathrm{cor}}{ }^{\mathrm{b}} \\
(\mathrm{au})\end{array}$ & $\begin{array}{c}E_{\mathrm{B} 3 \mathrm{LYP}}{ }^{\mathrm{c}} \\
(\mathrm{au})\end{array}$ & $\begin{array}{c}E_{\mathrm{MP} 2}{ }^{\mathrm{d}} \\
(\mathrm{au})\end{array}$ & $\begin{array}{c}H^{e} \\
(\mathrm{au})\end{array}$ & $\begin{array}{c}\mathrm{HOF} \\
\left(\mathrm{kJ} \cdot \mathrm{mol}^{-1}\right)\end{array}$ \\
\hline $\mathrm{CH}_{4}$ & 0.044793 & 0.048605 & -40.5261442 & -40.3796224 & -40.33280912 & $-74.9^{\mathrm{f}}$ \\
$\mathrm{CH}_{3} \mathrm{CH}_{3}$ & 0.074599 & 0.079027 & -79.8416413 & -79.5716299 & -79.49558686 & $-84.0^{\mathrm{f}}$ \\
Furoxan & 0.049510 & 0.054821 & -337.2249822 & -336.4521556 & -336.399315 & $225.3^{\mathrm{g}}$ \\
Tetrazole & 0.046860 & 0.051289 & -258.2680432 & -257.6538576 & -257.6044436 & $331.3^{\mathrm{g}}$ \\
BTTFO & 0.163919 & 0.185740 & -1523.4059122 & -1519.9902246 & -1519.8110416 & 1380.1
\end{tabular}

${ }^{\mathrm{a}}$ Zero-point correction; ${ }^{\mathrm{b}}$ Thermal correction to Enthalpy; ${ }^{\mathrm{c}}$ Total energy (B3LYP); ${ }^{\mathrm{d}}$ Total energy (MP2); ${ }^{\mathrm{e}} H=E_{\mathrm{MP} 2}+H_{\mathrm{cor}}-(1-$ 0.96)ZPE; ${ }^{\mathrm{f}}$ Data from NIST; ${ }^{\mathrm{g}}$ Calculated by atomization at the CBS-4M level.

The solid-state enthalpy of formation for a neutral compound can be estimated by subtracting the heat of sublimation from gas phase heat of formation. On basis of the literatures ${ }^{[8,9]}$, the heat of sublimation can be estimated with Trouton's rule according to eq 1 , where $\mathrm{T}$ represents either the melting point or the decomposition temperature when no melting occurs prior to decomposition:

$\Delta H_{\text {sub }}=188 / \mathrm{J} \mathrm{mol}^{-1} \mathrm{~K}^{-1} \times T$

\section{Cartesian Coordinates of Optimized geometries}<smiles></smiles>

$\begin{array}{llll}\mathrm{O} & 2.22392 & 2.079254 & -1.98442 \\ \mathrm{O} & -0.254102 & -2.78503 & -0.387843 \\ \mathrm{O} & -1.680164 & 2.518857 & 0.984774 \\ \mathrm{O} & 1.905138 & -2.649916 & -1.134268 \\ \mathrm{~N} & 1.211268 & 1.200102 & -1.853491 \\ \mathrm{~N} & 3.319445 & 1.67701 & -1.071836 \\ \mathrm{~N} & 0.91353 & -2.033336 & -0.75669 \\ \mathrm{~N} & -1.215113 & -1.902868 & -0.000155 \\ \mathrm{~N} & -0.801987 & 1.56531 & 0.64312 \\ \mathrm{~N} & -3.054367 & 1.967532 & 0.829984 \\ \mathrm{C} & 2.880754 & 0.572161 & -0.448743\end{array}$




\begin{tabular}{llll}
\hline $\mathrm{C}$ & 1.571082 & 0.314036 & -0.955777 \\
$\mathrm{C}$ & 0.627721 & -0.74089 & -0.582488 \\
$\mathrm{C}$ & -0.719338 & -0.691522 & -0.103779 \\
$\mathrm{C}$ & -1.477357 & 0.49392 & 0.296526 \\
$\mathrm{C}$ & -2.892276 & 0.702124 & 0.401193 \\
$\mathrm{O}$ & 4.325531 & 2.344357 & -1.054591 \\
$\mathrm{O}$ & -3.986041 & 2.684458 & 1.092655 \\
$\mathrm{C}$ & 3.677713 & -0.097611 & 0.563933 \\
$\mathrm{~N}$ & 3.816034 & -1.43848 & 0.689976 \\
$\mathrm{~N}$ & 4.374415 & 0.482058 & 1.529138 \\
$\mathrm{~N}$ & 4.611078 & -1.684217 & 1.748051 \\
$\mathrm{H}$ & 3.452228 & -2.189665 & 0.10748 \\
$\mathrm{~N}$ & 4.936485 & -0.528916 & 2.236568
\end{tabular}<smiles>NC(=C1C(c2nnn[nH]2)=NON1O)N1NNN1</smiles>

$\begin{array}{llll}\mathrm{N} & 1.092288 & 1.988782 & 0.662795 \\ \mathrm{C} & 0.397811 & 0.909137 & 0.317198 \\ \mathrm{C} & 1.322028 & -0.123783 & 0.132581 \\ \mathrm{~N} & 2.578055 & 0.301958 & 0.294709 \\ \mathrm{O} & 2.482927 & 1.650312 & 0.647995 \\ \mathrm{C} & -1.109283 & 0.912986 & 0.167983 \\ \mathrm{C} & 1.021538 & -1.584741 & -0.202883 \\ \mathrm{~N} & -1.737432 & 0.113409 & -0.660626 \\ \mathrm{~N} & -3.068467 & 0.440154 & -0.547515 \\ \mathrm{~N} & -3.245108 & 1.433156 & 0.315761 \\ \mathrm{~N} & -2.018659 & 1.735936 & 0.763385 \\ \mathrm{~N} & 1.065934 & -2.552960 & 0.682216 \\ \mathrm{~N} & 0.730109 & -3.696164 & -0.007436 \\ \mathrm{~N} & 0.475522 & -3.427648 & -1.282733 \\ \mathrm{~N} & 0.645763 & -2.103197 & -1.401697 \\ \mathrm{O} & 0.777815 & 3.155229 & 0.990468 \\ \mathrm{H} & -1.891037 & 2.498993 & 1.419698 \\ \mathrm{H} & 0.480196 & -1.651561 & -2.291900\end{array}$

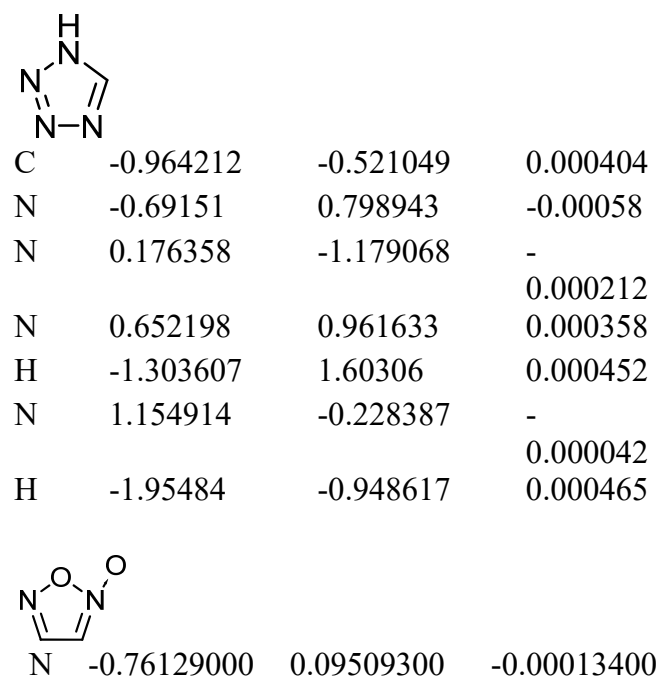




\begin{tabular}{clll}
\hline $\mathrm{N}$ & 1.38213500 & -0.71964000 & -0.00004400 \\
$\mathrm{O}$ & -1.97612900 & -0.03902100 & 0.00007300 \\
$\mathrm{O}$ & 0.07236400 & -1.10666000 & 0.00003300 \\
$\mathrm{C}$ & 0.08240800 & 1.12243100 & 0.00004000 \\
$\mathrm{C}$ & 1.39182200 & 0.59012600 & 0.00002100 \\
$\mathrm{H}$ & 2.32894700 & 1.12875000 & 0.00025600 \\
$\mathrm{H}$ & -0.29012300 & 2.13318000 & -0.00021200 \\
& & & \\
$\mathrm{CH}_{3} \mathrm{CH}_{3}$ & & \\
$\mathrm{C}$ & 0.00000000 & 0.00000000 & 0.76604800 \\
$\mathrm{H}$ & 0.50997400 & 0.88394300 & 1.16513600 \\
$\mathrm{H}$ & 0.51053000 & -0.88362200 & 1.16513600 \\
$\mathrm{H}$ & -1.02050400 & -0.00032100 & 1.16513600 \\
$\mathrm{C}$ & 0.00000000 & 0.00000000 & -0.76604800 \\
$\mathrm{H}$ & 1.02050400 & -0.00032100 & -1.16513600 \\
$\mathrm{H}$ & -0.51053000 & -0.88362200 & -1.16513600 \\
$\mathrm{H}$ & -0.50997400 & 0.88394300 & -1.16513600 \\
& & & \\
$\mathrm{CH}$ & & & \\
$\mathrm{C}$ & 0.00000000 & 0.00000000 & 0.00000000 \\
$\mathrm{H}$ & 0.63088200 & 0.63088200 & 0.63088200 \\
$\mathrm{H}$ & -0.63088200 & -0.63088200 & 0.63088200 \\
$\mathrm{H}$ & -0.63088200 & 0.63088200 & -0.63088200 \\
$\mathrm{H}$ & 0.63088200 & -0.63088200 & -0.63088200
\end{tabular}

\section{References}

[1] Frisch, M. J.; Trucks, G. W.; Schlegel, H. B.; Scuseria, G. E.; Robb, M. A.; Cheeseman, J. R.; Montgomery Jr, J. A.; Vreven, T.; Kudin, K. N.; Burant, J. C.; Millam, J. M.; Iyengar, S. S.; Tomasi, J.; Barone, V.; Mennucci, B.; Cossi, M.; Scalmani, G.; Rega, N.; Petersson, G. A.; Nakatsuji, H.; Hada, M.; Ehara, M.; Toyota, K.; Fukuda, R.; Hasegawa, J.; Ishida, M.; Nakajima, T.; Honda, Y.; Kitao, O.; Nakai, H.; Klene, M.; Li, X.; Knox, J. E.; Hratchian, H. P.; Cross, J. B.; Bakken, V.; Adamo, C.; Jaramillo, J.; Gomperts, R.; Stratmann, R. E.; Yazyev, O.; Austin, A. J.; Cammi, R.; Pomelli, C.; Ochterski, J. W.; Ayala, P. Y.; Morokuma, K.; Voth, G. A.; Sal-vador, P.; Dannenberg, J. J.; Zakrzewski, V. G.; Dapprich, S.; Daniels, A. D.; Strain, M. C.; Farkas, O.; Malick, D. K.; Rabuck, A. D.; Raghavachari, K.; Foresman, J. B.; Ortiz, J. V.; Cui, Q.; Baboul, A. G.; Clifford, S.; Cioslowski, J.; Stefanov, B. B.; Liu, G.; Liashenko, A.; Piskorz, P.; Komaromi, I.; Martin, R. L.; Fox, D. J.; Keith, T.; Al-Laham, M. A.; Peng, C. Y.; Nanayakkara, A.; Challacombe, M.; Gill, P. M. W.; Johnson, B.; Chen, W.; Wong, M. W.; Gonzalez, C and Pople, J. A. Gaussian 09, Revision A. 02; Gaussian, Inc., Wallingford, CT, 2009.

[2] Dennington, T. K. R. Millam J. GaussView 5, V5.0.8, Semichem Inc., Shawnee Mission, 2009.

[3] Parr, R. G.; Yang, W. Density Functional Theory of Atoms and Molecules, Oxford University Press, New York, 1989.

[4] Head-Gordon, M.; Pople. J. A. MP2 Energy Evaluation by Direct Methods. Chem. Phys. Lett. 1988, 153, $503-506$.

[5] Ochterski, J. M.; Petersson, G. A.; Montgomery. J. A. A Complete Basis Set Model Chemistry. V. Extensions to Six or More Heavy Atoms. J. Chem. Phys. 1996, 104, 2598-2619.

[6] Montgomery, J. A.; Frisch, M. J.; Ochterski, J. M.; Petersson, G. A. A Complete Basis Set Model Chemistry. VII. Use of the Minimum Population Localization Method. J. Chem. Phys. 2000, 112, 6532-6542.

[7] Linstrom, P. J.; Mallard, W. G. NIST Chemistry WebBook, NIST Standard Reference Database, 69, National Institute of Standards and Technology, 2005.

[8] Trouton, F. IV. On Molecular Latent Heat. Philos. Mag. 1884, 18, 54-57.

[9] Westwell, M. S.; Searle, M. S.; Wales, D. J.; Wiliams. D. H. Empirical Correlations between Thermodynamic Properties and Intermolecular Forces. J. Am. Chem. Soc., 1995, 117, 5013-5015. 